\title{
Der Betrieb als Ort Lebensbegleitenden Lernens?
}

Winfried Heidemann

Vorliegende Makrostudien vermitteln uns überwiegend das Bild, dass die betriebliche Weiterbildung in Deutschland ein nur niedriges quantitatives Niveau erreicht. Über diese Feststellung hinaus fehlt es an Informationen über die betriebliche Weiterbildungspraxis. Jedoch gerade dort tut sich mehr und anderes, als es die Datensätze solcher Studien erfassen können. Vor dem Hintergrund von Erfahrungen in der Beratungstätigkeit für Betriebsräte geht der Beitrag den Ansätzen informellen und formellen Lernens und der Ressourcenorganisation in Betrieben nach. Damit wollen wir zur Diskussion darüber beitragen, ob und wie in der betrieblichen Praxis vorhandene Formen weiterentwickelt werden können, um den Betrieb zu einem Ort lebenslangen Lernens zu machen.

\section{WEITERBILDUNG IM BETRIEB: DAS VORHERRSCHENDE BILD}

Daten zur Situation betrieblicher Weiterbildung liegen aus verschiedenen wissenschaftlichen Untersuchungen vor. Die Ergebnisse von Befragungen mit repräsentativem Anspruch sind hinsichtlich der Beteiligung von Betrieben und Beschäftigten an Weiterbildung allerdings widersprüchlich. Das ist vor allem methodisch bedingt, weil die Erhebungszeitpunkte und -zeiträume, die einbezogenen Weiterbildungsoder Lernformen, die jeweils Befragten Betrieb oder Unternehmen, Management oder Beschäftigte - und die Betriebs- oder Unternehmensgrößen in den Stichproben teilweise stark differieren. Ein Überblick über vier nationale und eine internationale Untersuchung aus den 2000er Jahren zeigt: Die Quote der Betriebe mit Weiterbildung reicht von $45 \%$ bis $100 \%$, die der Nutzung von Weiterbildung durch Beschäftigte liegt zwischen $25 \%$ bei formaler Weiterbildung und $91 \%$ bei informeller Weiterbildung von Fachangestellten. ${ }^{1}$ Doch es gibt auch
Übereinstimmungen: Alle Untersuchungen belegen die Abhängigkeit des Zugangs zu betrieblichem Lernen von Vorbildung, betrieblicher Statusposition, Branche und Betriebsgröße.

International vergleichende Untersuchungen sehen Deutschland bei der Teilnahme an Bildung und beim Bildungserfolg stets abgeschlagen. Das gilt auch für die betriebliche Weiterbildung, wie die alle sechs (in Zukunft fünf) Jahre wiederholte große europäische Untersuchung Continuous Vocational Training Survey (CVTS) in Unternehmen der Privatwirtschaft mit mehr als 20 Beschäftigten zeigt. ${ }^{2}$ Hiernach ist die Beteiligung von Unternehmen und Beschäftigten an förmlicher Weiterbildung in fast allen west- und nordeuropäischen Industriestaaten in der ersten Hälfte der 2000er Jahre zurückgegangen. Deutschland liegt in dieser Ländergruppe am Ende, in einem Vergleich mit allen europäischen Staaten rückt Deutschland in das untere Mittelfeld.

Wegen unterschiedlicher Erhebungsmethoden und Rückläufe dieser Untersuchung in den beteiligten Ländern ist ein Vergleich der Zahlen allerdings nur begrenzt möglich. ${ }^{3}$ Dessen ungeachtet gibt CVTS Hinweise auf einen unterschiedlichen Stellenwert der verschiedenen Angebotsformen der Weiterbildung in deutschen Unternehmen im Vergleich zu Unternehmen in anderen europäischen Ländern. Es erweist sich als sinnvoll, zwischen formaler und informeller Weiterbildung im Betrieb zu differenzieren. ${ }^{4}$

\section{FORMALES UND INFORMELLES LERNEN IM BETRIEB}

Geht es nämlich um förmliche Weiterbildungskurse - egal ob sie im Unternehmen selbst oder außerhalb durchgeführt werden - dann liegen nach CVTS deutsche Unternehmen mit einer Beteiligungsrate von $54 \%$ (2005) deutlich hinter Schweden (72\%), Frankreich (71\%) sowie den Niederlanden und Finnland (70 \%). Auch einige

1 Heidemann, W. (Hrsg.) (2010): Lebenslanges Lernen im Betrieb - Neuere Praxisbeispiele. Arbeitspapier der Hans-Böckler-Stiftung 153, Düsseldorf (im Erscheinen).

2 Behringer, F./Moraal, D./Schönfeld, G. (2008): Betriebliche Weiterbildung in Europa: Deutschland weiterhin nur im Mittelfeld, Aktuelle Ergebnisse aus CVTS 3, in: Berufsbildung in Wissenschaft und Praxis (BWP) 1, S. 9-14

3 Die Untersuchung wurde in einigen Ländern mit schriftlichem Fragebogen, in anderen mit mündlichen Interviews, in dritten mit Online-Befragungen und in weiteren mit einem Methodenmix durchgeführt; auch die Rückläufe der Antworten sind sehr unterschiedlich: Deutschland liegt mit einer Rücklaufquote von 27 \% am unteren Ende, in anderen Ländern ist sie sehr viel höher, teilweise über $70 \%$. Schließlich sind die Rückläufe für die einzelnen Unternehmensgrößenklassen ebenfalls sehr unterschiedlich. In einigen Ländern liegt die Beteiligung von kleinen und Mittelunternehmen an der Befragung höher als die von Großunternehmen, in anderen Ländern ist es genau umgekehrt. (Informationen des Autors dieser Studie auf einem Workshop zur Vorbereitung von CVTS 4 im Juni 2009 am Europäischen Berufsbildungszentrum Cedefop in Thessaloniki.)

4 Hier ist die Begrifflichkeit uneinheitlich: Neben der Unterscheidung zwischen "formal learning “ (als Teil eines Bildungsganges im Bildungssystem), "non-formal learning" (intentional betriebenes Lernen in Kursen oder Veranstaltungen, die nicht Teil eines Bildungsganges sind) und "informal learning " ( Nebenbei-Lernen“ im Arbeits- oder Lebensprozess) gibt es die zwischen "formaler Weiterbildung" (egal, ob im Rahmen eines Bildungsgangs oder im Betrieb) und "informeller Weiterbildung".

Winfried Heidemann, Dr., Referatsleiter Qualifikation in der Abteilung Mitbestimmungsförderung der Hans-Böckler-Stiftung Arbeitsschwerpunkte: Analysen und Institutionenberatung zu Lebenslangem Lernen, Beschäftigungsfähigkeit, Beschäftigungssicherung, Sozialer Unternehmensverantwortung. e-mail:Winfried-Heidemann@boeckler.de 
der ostmitteleuropäischen Beitrittstaaten liegen vor Deutschland. Dabei ist die Beteiligung der Unternehmen gegenüber 1999 in allen west- und nordeuropäischen Ländern zum Teil deutlich zurückgegangen: In Deutschland um 13 Prozentpunkte und in den früheren „Vorzeigeländern“ um zwölf (Niederlande), elf (Schweden) oder fünf Prozentpunkte (Finnland).

Ähnlich sieht es nach dieser Erhebung bei der Beteiligungsrate der Beschäftigten an Weiterbildungskursen im betrieblichen Kontext aus. Hier ist die Beteiligung in Deutschland zwischen 1999 und 2005 von $32 \%$ auf $30 \%$ aller Beschäftigten abgesunken. Noch stärkere Rückgänge sind in Schweden, Finnland und den Niederlanden zu verzeichnen. Dennoch liegt die Weiterbildungsquote der Arbeitnehmer in diesen Ländern mit $46 \%$, $39 \%$ und $34 \%$ deutlich vor der der deutschen Arbeitnehmer.

Ein gegenteiliges Bild ergibt sich, wenn die CVTS-Untersuchung neben Kursen "andere Formen der Weiterbildung" (arbeitsplatznahe Qualifizierung, selbstgesteuertes Lernen etc.) betrachtet. Hier liegt Deutschland mit $66 \%$ der Unternehmen, die solche Weiterbildungsformen anbieten, unmittelbar hinter Österreich und vor Schweden (60\%), Finnland (56\%), Niederlanden (52\%) und Frankreich (44\%). Allerdings muss auch mit Blick auf die „anderen“ Formen der Weiterbildung konstatiert werden, dass die Wahrnehmung solcher Angebote seitens der Unternehmen seit 1999 zum Teil deutlich zurückgegangen ist (in Deutschland von $72 \%$ auf $66 \%$ ).

Diese Ergebnisse können Anzeichen für eine in deutschen Betrieben im Vergleich zu anderen Ländern unterschiedliche Art von Weiterbildung sein. Das kann mit der Organisation der betrieblichen Leistungserstellung oder mit der spezifischen beruflichen Ausbildung der Beschäftigten zusammenhängen, die zu anderen Formen der Weiterbildung führen. Die europäische Befragung gibt dazu keine Erklärung. Hinweise gibt es jedoch darauf, dass Kurse und andere Formen nicht in einer Substitutionsbeziehung stehen, sondern sich ergänzen. ${ }^{5}$

$\mathrm{Zu}$ Deutschland kommt eine repräsentative Untersuchung des Zentrums für Sozialforschung Halle (zsh), ${ }^{6}$ die 2002 in Betrieben der Privatwirtschaft mit 20 bis 1.000 Beschäftigten durchgeführt worden ist, zu aufschlussreichen Ergebnissen. Sie unterscheidet neben formaler Weiterbil- dung zwischen dem traditionellen informellen Lernen und den neuen Formen informellen Lernens:

(1) $\mathrm{Zu}$ den traditionellen informellen Lernformen gehören Weiterbildung am Arbeitsplatz, Unterweisung durch Vorgesetzte, Teilnahme an Fachvorträgen, Tagungen und Informationsveranstaltungen.

(2) Neue Formen informellen Lernens sind Teamarbeit, Qualitätszirkel, Wissenstransfer durch Kollegen, Jobrotation, Partnerschaften, Coaching und auch computergestütztes Lernen.

Die Untersuchung des zsh nimmt die unterschiedlichen Formen des Lernens vor dem Hintergrund betrieblicher Innovationsprozesse in den Blick. Sie fragt, ob Betriebe und Beschäftigte überhaupt solche Lernformen anbieten und nutzen, und sieht von einem genauen zeitlichen Bezugsraum ab. Auf dieser Grundlage lautet der Befund: In Deutschland nutzen weit mehr Betriebe als bislang vermutet eine dieser Lernformen (formales Lernen $81 \%$, traditionelles informelles Lernen $92 \%$, neue informelle Lernformen $74 \%$ der Betriebe). Dabei bestehen die Unterschiede zwischen den betrieblichen Statusgruppen bei der Beteiligung an betrieblichen Lernformen auf höherem Niveau fort: Von den An- und Ungelernten nehmen an formalem Lernen $47 \%$, an traditionellen informellen Lernformen $64 \%$ und an neuen informellen Lernformen $70 \%$ teil; für Fachangestellte lauten die Zahlen 84 \%, $91 \%$ und $93 \%$.

Nach der Untersuchung des zsh sind Art und Intensität der betrieblichen Aktivitäten in der Weiterbildung kontextabhängig und werden eher durch den Bedarf an Kompetenzentwicklung im Zusammenhang mit organisatorischen und technischen Innovationen sowie der Personalrekrutierung als durch die jeweils aktuelle Ausstattung des Betriebs mit finanziellen Ressourcen bestimmt.?

\section{DIE BETRIEBLICHE PRAXIS: EINE DIFFERENZIERTE WIRKLICHKEIT}

Die Erkenntnisse der Makroanalysen über das quantitative Ausmaß betrieblicher Weiterbildungsaktivitäten sind wichtig, erfassen aber nur einen Teil der Wirklichkeit. Ein anderer zeigt sich, wenn wir näher in die Praxis des betrieblichen Lernens hineinschauen. In unserer Beratungstätigkeit für Betriebsräte sehen wir neben Defiziten betrieblicher Weiterbildung eine vielfältige Praxis des Lernens im Arbeitszusammenhang, an deren Entfaltung Betriebsräte vor allem in Groß- und Mittelbetrieben einen Anteil haben. Dies zeigen 22 Kurzfallstudien, die wir in den letzten Jahren im Kontext unserer Beratungstätigkeit und für Beratungszwecke erstellt haben. ${ }^{8}$ Sie kommen aus unterschiedlichen Branchen, aus der gewerblichen Wirtschaft wie aus dem Dienstleistungsbereich, es überwiegen Großbetriebe mit mehr als 2.000 Beschäftigten, aber auch Mittelunternehmen sind dabei. Die Fallstudien kamen nicht durch systematische Auswahl zustande, sondern ergaben sich aus Beratungsanfragen und neueren Betriebsvereinbarungen. Sie erheben damit keinen repräsentativen Anspruch, sondern bieten „informierte Einblicke" in das betriebliche Geschehen, die unser Wissen verbreitern und differenzieren und auch Fragestellungen für weitere Forschung generieren können. Sie dokumentieren drei „Stränge“: eine verbreitete Praxis der informellen betrieblichen Kompetenzentwicklung, einzelne Ansätze der Verbindung von Kompetenzentwicklung mit formalen Qualifikationen sowie die differenzierte Nutzung der verschiedenen Ressourcen für Lebensbegleitendes Lernen.

Deutlich wird: Bei betrieblicher Kompetenzentwicklung wird Lebenslanges Lernen prozesshaft. Die für betriebliche Innovationen notwendige Qualifizierung zur Anpassung und Entwicklung der Kompetenzen wird meistens - wenn auch mit Friktionen - organisiert. Schwachstellen scheinen weniger in der betrieblichen Kompetenzentwicklung denn eher in der Weiterbildung für persönliche Beschäftigungsfähigkeit und individuellen Bedarf der Beschäftigten zu liegen. Weiterhin zeigt sich: Weiterbildung ist ein „Managementthema". Inwieweit der Betriebsrat eine aktive Rolle spielt, ist stark abhängig von der Mitbestimmungskultur im Unternehmen.

5 Behringer, F./Descamps, R. (2009): Determinants of employer-provided training: a comparative analysis of Germany and France, in: ZBW, Beiheft 22, S. 93-123.

6 Winge, S./Wiener, B. (2009): Lernen in kleinen und mittleren Unternehmen. Forschungsberichte aus dem zsh 09-1.

7 Lutz, B. (2005): Organisierte Weiterbildung und informelles Lernen im Überblick, in: Winge, $S$. (Hrsg.): Kompetenzentwicklung in Unternehmen. Forschungsberichte aus dem zsh 05-1, S. 128ff.

8 Online-Veröffentlichung der Fallstudien: www. boeckler.de - Mitbestimmungsförderung - Personal und Bildung (Zugriff 04.02.2010). 


\section{FORMEN DER KOMPETENZ- ENTWICKLUNG}

Die ständige Weiterentwicklung der Kompetenzen der Beschäftigten für die betriebliche Leistungserstellung ist in den letzten Jahren zum vorrangigen Thema der betrieblichen Weiterbildung geworden. ${ }^{9}$ Mit dem Konzept betrieblicher Kompetenzentwicklung kommen nicht allein die fachlichen, sondern auch überfachliche, methodische und soziale Kenntnisse und Fertigkeiten in den Blick. Neben formalen Kursen werden verstärkt arbeitsplatz- und arbeitsprozessnahe Formen der Qualifizierung genutzt, Arbeiten und Lernen werden entgrenzt. Eine Auswertung von einschlägigen Betriebsvereinbarungen des Archivs in der Hans-Böckler-Stiftung zeigt Regelungen zu den hierfür einschlägigen Instrumenten auf: Mitarbeitergespräch, Potenzialanalyse, Training on the Job und Job-Rotation. ${ }^{10}$

Unsere Betriebsfallstudien enthalten aber auch Beispiele, wie Anlernprozesse am Arbeitsplatz mit Formen des Lernens außerhalb der Arbeit und mit Lernangeboten für die individuelle Entwicklung verbunden werden und dies zur Vorbereitung auf formale Bildungsabschlüsse genutzt werden kann. Die Regelungen in Betriebsvereinbarungen, persönliche Entwicklungsziele in Mitarbeitergespräche aufzunehmen und Lernergebnisse zu zertifizieren, zeigen die Möglichkeit, kontinuierliche Kompetenzentwicklung durch die Förderung von Lernbereitschaft und Engagement der Beschäftigten abzusichern. Allerdings ist die modulare Zusammensetzung von betrieblichen Qualifizierungsschritten zu anerkannten beruflichen Abschlüssen bisher noch die Ausnahme.

\section{LERNRESSOURCEN}

Lernen braucht Ressourcen. ${ }^{11}$ Im Vordergrund der Debatten stehen dabei zumeist Zeit und Geld. Unsere Auswertungen von Betriebsvereinbarungen zur betrieblichen Weiterbildung von Ende der 1990er und Mitte der 2000er Jahre zeigen Regelungen zum Einsatz dieser Ressourcen. ${ }^{12}$ Verbreitet sind Verabredungen über eine Kostenteilung, zumindest wenn es um Lernen für die persönliche Entwicklung geht. Üblich ist in solchen Fällen, dass Beschäftigte Freizeit für Weiterbildung oder auch zusätzliche unbezahlte wöchentliche Lernzeiten einbringen. ${ }^{13}$ Das Konzept betrieblicher Lernzeitkonten, auf denen Mehrarbeit angesammelt wird, die für Weiterbildung genutzt wird, hat sich in der betrieblichen Praxis jedoch nicht durchgesetzt. Auch dort, wo es Arbeitszeitkonten gibt, die das prinzipiell ermöglichen, werden sie nicht für betriebliches Lernen genutzt. ${ }^{14}$

Und ohnehin: Anders als es die Weiterbildungsdiskussion vermuten lässt, spielen in betrieblichen Vereinbarungen statt Zeit und Geld häufig ganz andere Ressourcen eine große Rolle. ${ }^{15}$ Als in der Praxis ebenso wichtig haben sich die Ressourcen Motivation, Organisation, Qualität und Anerkennung herausgestellt. Diese Entwicklung scheint sich in den letzten Jahren verstärkt zu haben. Damit Beschäftigte Bildungsangebote in Anspruch nehmen, müssen sie zur Beteiligung motiviert sein; eine geeignete Organisation von Bildungsprozessen und die Sicherstellung von Qualität der Angebote und ihrer Durchführung sind nötig zur Sicherung der Effektivität von Weiterbildung; und die Ressource „Anerkennung“von Bildungsergebnissen in Form von Zertifikaten, Abschlüssen, verbesserten Arbeitsbedingungen oder Bezahlung ist erforderlich, um die Beteiligungsbereitschaft sicherzustellen.

Unsere Betriebsfallstudien offenbaren eine differenzierte Kombination und Nutzung der verschiedenen Ressourcenarten. Zumeist werden mehrere Ressourcen genutzt. Auf der Angebotsseite steht die Organisation im Vordergrund: Eine gute Organisation des Lernens im Betrieb kann durch keine andere Ressource ersetzt werden. Auf der Nachfrageseite, bei den Beschäftigten, ist die Ressource Motivation unverzichtbar, um Beteiligung zu gewährleisten und Lernen effektiv werden zu lassen. In 16 unserer Fallstudien spielt die Ressource Organisation, in jeweils zehn Motivation und Zeit und in neun die Ressource Geld eine Rolle.

Das komplexe Verhältnis der unterschiedlichen Ressourcen und von informellen und formalen Lernangeboten zueinander illustriert das aktuelle Thema „Weiterbildung während Kurzarbeit“. Im Krisenjahr 2009 wurde diese Verbindung als Mittel angesehen, nicht nur Entlassungen zu verhindern, sondern auch die Kompetenzen der Beschäftigten für den Betrieb zu optimieren. Die Konjunkturpakete der Bundesregierung haben die Förderbedingungen dafür ausgeweitet und verbessert: Diese Möglichkeiten wurden aber nur selten genutzt: Weniger als 1\% der Kurzarbeiter wurden in Qualifizierung einbezogen, die durch Mittel des Europäischen Sozial- fonds und der Bundesagentur für Arbeit gefördert waren. ${ }^{16}$ Das wirft die Frage auf, woran es trotz der guten Anreize gefehlt hat.

Erste vorläufige Antworten liefern gegebenenfalls unsere betrieblichen Beispiele aus der Stahl- und Glasindustrie. Sie weisen auf, wie betriebliche Kompetenzentwicklung durch informelles und kursförmiges Lernen und die verschiedenen weiteren und als notwendig erachteten Ressourcen miteinander verbunden werden: Die betriebliche Ressource der Kurzarbeit, externe finanzielle Ressourcen der Bundesagentur für Arbeit, die Förderung von Motivation und Anerkennung bei den Beschäftigten sowie die Nutzung betrieblicher Organisationsstrukturen für eine zielorientierte Lernorganisation. In unseren Beispielen wurde durch Betriebsvereinbarung das Kurzarbeitergeld bei Beteiligung an Weiterbildung aufgestockt. Themen und Inhalte der Qualifizierungsmaßnahmen knüpfen an Analysen des Qualifizierungsbedarfs und an vorhandene Weiterbildungsprogramme des Betriebs an oder wurden unter Beteiligung der Kurzarbeitenden weiterentwickelt. Ergebnis sind sowohl Qualifizierungen am Arbeitsplatz als auch innerbetriebliche Kurzseminare und nach AZWV (Aner-

9 Siehe die Beiträge in Grote, S./Kauffeld, S./Frieling, E. (Hrsg.) (2006): Kompetenzmanagement, Grundlagen und Praxisbeispiele, Stuttgart.

10 Schwarzmeier, A. (2009): Betriebliche Kompetenzentwicklung: Regelungen in Betriebsvereinbarungen, Düsseldorf. Online-Veröffentlichung: http://www.boeckler.de/pdf/mbf_bvd_kompetenzentwicklung.pdf (Zugriff am 04.02.2010).

11 Zum Ressourcenkonzept - mit allerdings et was anderer Zuordnung - siehe Hummelsheim S. (2010): Finanzierung der Weiterbildung in Deutschland, Bielefeld, S. 13.

12 Heidemann, W. (1999): Betriebliche Weiterbildung, Düsseldorf, Edition der Hans-BöcklerStiftung 13, Düsseldorf; Busse, G./Heidemann, W. (2005): Betriebliche Weiterbildung, Reihe Betriebs- und Dienstvereinbarungen, Frankfurt.

13 Heidemann, W. (1999): Betriebliche Weiterbildung, Edition der Hans-Böckler-Stiftung 13, Düsseldorf, S. 26ff.

14 Heidemann, W. (2009): Bildungszeitkonten Betriebliche Verbreitung und Beispiele, in: WSIMitteilungen 8, S. 453-455

15 Anders: Busse, G./Seifert. H. (2009): Tarifliche und betriebliche Regelungen zur beruflichen Weiterbildung, Edition der Hans-Böckler-Stiftung 233, Düsseldorf, S. 67ff. Sie kommen auf schmalerer quantitativer Basis, aber mit weiter zurückreichendem Zeithorizont zum Ergebnis einer höheren Bedeutung der Kostenressourcen.

16 Heidemann, W. (2009): Qualifizierung in Kurzarbeit. Online-Veröffentlichung: http://www. boeckler.de/pdf/mbf_pers_bild_quali_kurzarbeit. pdf (Zugriff am 04.02.2010). 
kennungs- und Zulassungsverordnung Weiterbildung der Bundesagentur für Arbeit) zertifizierte Qualifizierungsangebote externer Bildungsträger - alle von kürzerer Dauer (zwischen 15 und 37 Stunden).

Deutlich wird anhand solcher Positivbeispiele auch, warum die Verbindung von Kurzarbeit und Qualifizierung im großen Maßstab kaum aufgegriffen wurde: Wegen der unterschiedlichen zeitlichen und organisatorischen Perspektiven und Logiken von Kurzarbeit und Weiterbildung ist eine Verbindung nur schwer möglich. Voraussetzung ist eine bereits vorhandene Weiterbildungskonzeption und eine Weiterbildungskultur im Betrieb. Zeit und Geld allein reichen nicht. Die aufgezeigten positiven Fälle resultieren aus einer für betriebliche Qualifizierung förderlichen beteiligungsorientierten Unternehmenskultur. Das bedeutet aber auch: Die vielfach formulierte Annahme, die Betriebe verschöben in der Hochkonjunktur die nötige Weiterbildung und könnten sie nun während der Wirtschaftskrise ohne Weiteres nachholen, findet in der Praxis keine Bestätigung.

\section{QUALIFIZIERUNGSTARIFVERTRÄGE}

Die europäische Weiterbildungserhebung CVTS 3 hat Indizien für eine das Weiterbildungsengagement der Unternehmen fördernde Wirkung von Verträgen der Sozialpartner festgestellt. ${ }^{17}$ In Deutschland stehen in den Qualifizierungstarifverträgen mehrerer Branchen aus Anfang der 2000er Jahre regelmäßige individuelle Qualifizierungsgespräche und -vereinbarungen im Mittelpunkt. Effekte dieser Flächenverträge für eine erweiterte quantitative Teilnahme an betrieblicher Weiterbildung weisen die Makrountersuchungen bisher nicht aus. ${ }^{18}$ Unsere Fallbeispiele dazu machen deutlich, dass die Regelungen der Tarifverträge in der Umsetzung auf ein erhöhtes Engagement der Betriebsräte angewiesen sind und offenbar erst in längerfristiger Perspektive zu einer veränderten Kultur des Lernens in Betrieben beitragen können. Nach unseren Beobachtungen bestehen mögliche Effekte der Tarifverträge darin, dass gerade durch die Verfahren der Qualifizierungsgespräche die Tendenz zur betrieblichen Kompetenzentwicklung verstärkt wird, dafür aber in Betriebsvereinbarungen faire Bedingungen für die Beschäftigten festgelegt werden können. Die nach den Tarifverträgen ebenfalls mögliche Förderung persönlicher Weiterbildung ist nach unseren Erkenntnissen bisher auf Einzelfälle beschränkt und nicht in der Fläche verallgemeinerbar.

\section{OFFENE FRAGEN}

Der Fokuswechsel auf betriebliches Lernen und der Blick in die betrieblichen Mikrostrukturen zeigen eine andere, weiterreichende Beteiligung an arbeitsplatzbezogener Qualifizierung als in den Makrostudien erkennbar wird. Auch die Regeln des Betriebsverfassungsgesetzes erfassen diese Art der Wirklichkeit nicht, sie gehen nach wie vor vom Charakter der Weiterbildung als abgrenzbarer „Maßnahme“ aus.

Und selbst wenn die hier vorgestellten Praxiserfahrungen nicht verallgemeinerbar sind, so weisen sie uns doch auf in der Weiterbildungsdiskussion bislang vernachlässigte Aspekte und offene Fragen hin:

(1) Inwieweit kann die Entwicklung der Kompetenzen für betriebliche Zwecke auch die persönliche Beschäftigungsfähigkeit im Falle von Arbeitsplatz-, Betriebs- oder Berufswechsel stärken? Die Tarifverträge bieten durch die Berücksichtigung persönlicher Weiterbildungsinteressen in den Qualifizierungsgesprächen zwar ein Instrument dafür an, jedoch wird es in der Praxis bisher kaum umgesetzt.

(2) Wie kann die Anschlussfähigkeit des nicht-formalen und informellen betrieblichen Lernens an formale Bildung hergestellt werden? Chancen dafür können sich im Rahmen des kommenden Kreditpunktesystems für die berufliche Bildung entwickeln, in dem erworbene Kompetenzen bausteinartig zu Gesamtqualifikationen zusammengesetzt werden können.

(3) Und schließlich: Sollte der Betrieb - auch wenn er ein Ort für Lebenslanges Lernen ist, weil die Menschen dort einen großen Teil ihrer Lebenszeit verbringen - im Hinblick auf individuelle Beschäftigungsfähigkeit und persönliche Weiterentwicklung sinnvollerweise das Zentrum der Organisation von Lernangeboten sein? Hier sind Zweifel angebracht. Zumindest scheint es dafür nach wie vor der Ermöglichung von „personengetriebener" Weiterbildung in Lern- und Anerkennungszusammenhängen, die nicht auf den Einzelbetrieb beschränkt sind, zu bedürfen.

\footnotetext{
17 Ergebnisse von noch nicht veröffentlichten Zusatz auswertungen, mitgeteilt auf einem Workshop zur Vorbereitung von CVTS 4 im Juni 2009 bei Cedefop in Thessaloniki, dem Europäischen Zentrum für die Förderung der Berufsbildung.

18 Einen Überblick über Studien zur Praxis von Weiterbildungstarifverträgen geben Lenz, K./ Voß, A. (2009): Analyse der Praxiserfahrungen zum Qualifizierungstarifvertrag der Metall- und Elektroindustrie NRW, Arbeitspapier der HansBöckler-Stiftung 172, Düsseldorf.
} 